\title{
Cirugia de epilepsia en el Hospital Nacional Guillermo Almenara: primeros siete casos
}

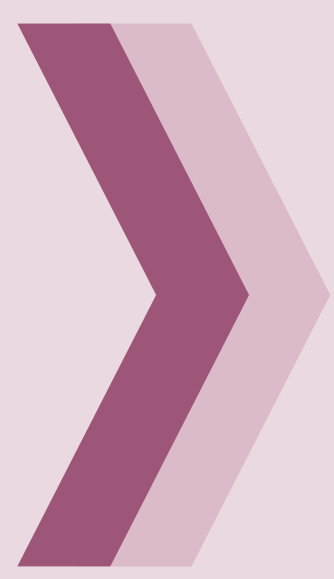

\section{Epilepsy surgery in Guillermo Almenara Hospital:} First seven cases

\section{Marco Antonio Mejía-Tupa, ${ }^{1}$ \\ Proel Pérez-Galdos, ${ }^{2}$ Alfredo Tori-Murgueytio, ${ }^{3}$ Mireya Del Solar-Tafur, ${ }^{4}$ William E. Anicama-Lima, ${ }^{5}$ Manuel G. Campos-Puebla ${ }^{6}$}

1. Neurocirujano. Magíster en Neurociencias. Departamento de Neurocirugía. Hospital Nacional Guillermo Almenara (HNGAI) EsSalud. Lima, Perú.

2. Neurólogo. Servicio de Especialidades Médicas. Hospital de Emergencias Miguel Grau EsSalud. Lima, Perú.

3. Neuropediatra. Departamento de Pediatría. HNGAI EsSalud. Lima, Perú.

4. Psicóloga. Servicio de Psicologia. HNGAI EsSalud. Lima, Perú

5. Anátomo patólogo. Servicio de Patología Quirúrgica. HNGAI EsSalud. Lima, Perú.

6. Neurocirujano. Departamento de Neurocirugía. Clínica Las Condes. Santiago, Chile.

\section{RESUMEN}

La epilepsia tiene una incidencia de 1 \% en la población, de los cuales un tercio no responde al tratamiento farmacológico, que conlleva a discapacidad y morbilidad secundaria. Los pacientes con epilepsia refractaria requieren un estudio multidisciplinario para el origen de la epilepsia y realizar la resección quirúrgica. En cinco pacientes del Hospital Almenara hicimos la evaluación clínica, neuropsicológica, estudio estructural con resonancia magnética, PET y SPECT y videoelectroencefalografía de cuero cabelludo $e$ intracraneal, con lo que realizamos la cirugía resectiva correspondiente. En $71 \%$ de los pacientes se logró la supresión completa de las crisis epilépticas y en $29 \%$ restantes, la disminución de la frecuencia en $75 \%$. La morbilidad adicional en los pacientes operados fue leve y manejable. El manejo multidisciplinario y tratamiento quirúrgico de la epilepsia refractaria en el hospital Almenara es altamente eficaz y se requiere la implementación de una unidad de Cirugía de Epilepsia.

Palabras claves: epilepsia; cirugía de epilepsia; resistencia a medicamentos; hemisferectomía; amigdalohipocampectomía; lobectomía; neuropsicología; tomografía por emisión de positrones; videoelectroencefalografía.

\begin{abstract}
Epilepsy has $1 \%$ prevalence, until one third are refractory to pharmacological treatment, so it produces disability, morbidity and mortality. These patients requires multidisciplinary diagnostic approach to localize the origin and ideally resect it. In Almenara Hospital, seven patients has been studied clinically, neuropsychologically, brain imaging: structure magnetic resonance and functional with positron emission tomography and single photon emission computed tomography and video electroencephalography non invasive initially and electrocorticography. After the study we decided and performed respective surgery. In $71 \%$ of patients we have got complete suppression of seizures and in the remain $29 \%$, more than $75 \%$ in seizure frequency. The side effects were mild and possible to treat. The multidisciplinary approach and surgical treatment of refractory epilepsy in the Almenara Hospital is highly efficient and it is needed to install an Specialized Unit.

KEYWORDs: epilepsy; medical resistant; surgery; treatment; outcomes; neuropsychology; hemispherectomy; lobectomy; positron emission tomography; video electroencephalography.
\end{abstract}




\section{INTRODUCCIÓN}

La epilepsia refractaria (ER) es una patología que produce una marcada discapacidad en los pacientes afectados, con un elevado riesgo de complicaciones e incluso de mortalidad por traumatismos, asfixia, ahogamiento o por la muerte súbita inesperada en pacientes con epilepsia ${ }^{1}$ (SUDEP, acrónimo de sudden unexpected death in epilepsy patients). Adicionalmente implica un alto costo económico para el paciente, familia, sociedad y el sistema de salud que lo atiende, que se ha estimado en 11 mil a 16 mil dólares anuales., ${ }^{2,3}$

La población mundial estimada para este año es de 7083 millones de habitantes, ${ }^{4}$ de los cuales $1 \%$ tienen epilepsia a nivel mundial según la $\mathrm{OMS}^{5}$ el decir 70,83 millones personas, de las cuales al menos $30 \%$ son refractarias al tratamiento farmacológico, ${ }^{6,7}$ es decir, 21,2 millones de personas afectadas con ER.

Considerando la población estimada en nuestro país en 31170724 habitantes para el presente año, ${ }^{8}$ el $1 \%$ estaría afectado de epilepsia, es decir 311707 habitantes, de los cuales solo $70 \%$ respondería adecuadamente a los fármacos antiepilépticos y el restante $30 \%$ es decir 93513 peruanos serían los afectados con ER.

En la Red Asistencial Almenara (RAA) de EsSalud, junto a la Oficina de Epidemiología hemos identificado en 34 meses de atención (enero 2008 a octubre 2010) a 2362 pacientes con diagnóstico de epilepsia; de los cuales, se deduce según los índices de prevalencia e incidencia internacionales, que al menos 709 pacientes con epilepsia en la RAA son refractarios al tratamiento médico.

La eficacia de la cirugía para el manejo de la ER es de $43 \%$ a $75 \%$ y la mortalidad relacionada a su aplicación es menor a 1 por 1000, ${ }^{9}$ por lo que la posibilidad de dar un alivio significativo a dichos pacientes es alta.

Hasta la fecha no hay publicaciones de cirugía de epilepsia realizada en nuestro país registradas en Medline o Lilacs, por lo que la tarea de implementar un programa de este tipo de estudio y tratamiento es capital para innovar y lograr un estándar de manejo similar a otros países de la región como Brasil, Chile, Argentina y Colombia.
El presente estudio describe la eficacia del enfoque multidisciplinario e innovador en el manejo de la epilepsia resistente al manejo farmacológico y los resultados obtenidos con el tratamiento quirúrgico de estos casos.

\section{MATERIAL Y MÉTODOS}

Se realizó un estudio descriptivo y retrospectivo en los pacientes con diagnóstico de epilepsia de la RAA que persisten con crisis epilépticas a pesar del uso de dos o más fármacos antiepilépticos y a quienes se les proporcionó tratamiento neuroquirúrgico.

Se consideraron 14 variables. El diagrama de variables se presenta en la Tabla 1.

\section{Definición operacional de variables}

- Eficacia: variable independiente, cualitativa, con escala ordinal, de medición indirecta a través de la disminución porcentual de la frecuencia de crisis epilépticas comparativa entre antes y después de la intervención quirúrgica, expresada según la escala de Engel, ${ }^{12}$

- Clase I. Libre de crisis incapacitantes: supresión total de crisis (al 100\%).

- Clase II. Crisis incapacitantes ocasionales; disminución de frecuencia de crisis entre $75 \%$ y $99 \%$.

- Clase III. Mejoría significativa; disminución de frecuencia de crisis de $74 \%$ a $50 \%$.

- Clase IV. Mejoría no significativa; disminución de frecuencia de crisis menor al $50 \%$.

- Edad de Inicio de epilepsia:variable moderadora, cuantitativa, con escala de razón, con medición directa a través de la edad cronológica expresada en años al momento de inicio de la epilepsia.

- Sexo: variable moderadora, cualitativa, de escala de medición nominal, de medición directa. Se expresó como masculino o femenino.

$\triangle$ Edad de cirugía: variable moderadora, cuantitativa, de escala de medición de razón, con medición directa según la fecha de nacimiento. Se expresó en años cumplidos a la fecha de la cirugía definitiva de epilepsia. 
^ Tiempo hasta la cirugía: variable moderadora, cuantitativa, con escala de razón, con medición indirecta a través del número de meses entre el inicio de la epilepsia y la fecha de la cirugía.

^ Número de focos epileptógenos: variable moderadora, cualitativa, con escala ordinal, de medición indirecta a través del número de focos de inicio de epilepsia mediante el uso de electroencefalografía o electrocorticografía de largo plazo. Podrá tener los valores de único y múltiple.

$\Delta$ Lado epileptógeno: variable moderadora, cualitativa, con escala nominal, de medición indirecta a través del informe de VEEG. Tiene los valores de derecho e izquierdo.

^ Lóbulo epileptógeno: variable moderadora, cualitativa, con escala nominal, de medición indirecta a través del informe de VEEG.

$\triangle$ Número de fármacos antiepilépticos (FAE) preoperatorios: variable moderadora, cuantitativa, con escala de medición intervalo. Se medirá en forma indirecta a través de la historia clínica preoperatoria. Tendrá los valores de dos, tres, cuatro o cinco FAE diferentes.

$\triangle$ Nivel cognitivo prequirúrgico: variable moderadora, de medición indirecta a través del coeficiente intelectual según la escala de Weschler, con escala de medición de intervalo. Tuvo los valores finales en categorías de promedio (90 a 109), medio bajo (80-89), retraso mental leve (70-79), retraso mental moderado (50-69) y retraso mental grave (20-49).

^ Tipo de cirugía: variable moderadora, de medición directa a través del informe operatorio, con escala de medición nominal. Tuvo los valores finales de:

- Electrocorticografía de largo plazo (EcoG LP) por tres días, seguida de una segunda cirugía con resección cortical focal.

- Electrocorticografía intraoperatoria (ECoG IO) por 30 minutos, con resección temporal lateral y medial.

- Hemisferectomía funcional en un solo tiempo.

- Diagnóstico definitivo: variable moderadora, cualitativa, con escala de medición nominal, que tuvo medición indirecta según los criterios clínicos y imagenológicos estructurales y funcionales, electrofisiológicos y estudio anatomopatológico. Tuvo los valores finales de displasia cortical focal, esclerosis del hipocampo y encefalitis de Rasmussen.

$\Delta$ Efectos adversos de la cirugía: variable moderadora, de medición indirecta según los reportes de evaluación clínica al alta de hospitalización y al último seguimiento. Con escala de medición ordinal, que tuvo los valores deausente-sino hubo ningún déficit neurológico añadido en el control final comparado a la evaluación preoperatoria- o permanente -si presentó algún efecto adverso o discapacidad luego de la intervención quirúrgica que persiste hasta el último control ambulatorio-.

^ Tiempo de seguimiento posquirúrgico: variable moderadora, de medición indirecta según la historia clínica, cuantitativa con escala de medición de razón. Expresado por el número de meses transcurridos entre la fecha de cirugía y el último control ambulatorio.

\section{Definición de términos}

\ Evaluación multidisciplinaria: valoración clínica realizada por los especialistas relacionados al manejo de la epilepsia, intervienen neurólogo, neuropediatra, neurocirujano y psicólogo, a través de la evaluación clínica, con test estructurados, resonancia magnética estructural y funcional (cuando lo amerita), tomografía de emisión de fotón simple (SPECT) tomografía de emisión de positrones (PET scan) y VEEG de largo plazo tanto a nivel de cuero cabelludo como a nivel cortical con electrodos subdurales.

- Tratamiento quirúrgico: consistió de dos fases, la diagnóstica y la terapéutica.

- Diagnóstica: en la cual se colocaron electrodos subdurales para registro intraoperatorio 0 ECoG IO, o de largo plazo o EcoG LP.

- Terapéutica: en las cuales se realizaron la resección o desconexión de las estructuras cerebrales identificadas como focos epileptogénicos: resección frontal parcial, lobectomía temporal o hemisferectomía funcional. 


\section{Auta Méäica Pernana}

\begin{tabular}{|c|c|c|c|c|c|}
\hline Variable & Tipo & Indicador (Es) & Método & Valores & $\begin{array}{l}\text { Escala de } \\
\text { medición }\end{array}$ \\
\hline$\Delta$ Eficacia & Independiente & $\begin{array}{l}\text { Escala de } \\
\text { Engel modificada }\end{array}$ & Indirecto & $\begin{array}{l}\text { Disminución \% frecuencia de crisis: } \\
\text { I al } 100 \% \text {, II } 99 \text { al } 75 \% \text {, } \\
\text { III } 50 \text { al } 74 \% \text { IV menor a } 50 \%\end{array}$ & Ordinal \\
\hline $\begin{array}{l}\text { Edad de inicio } \\
\text { de epilepsia }\end{array}$ & Moderadora & Edad cronológica & Directo & $\begin{array}{l}\text { Número de años al inicio de la } \\
\text { epilepsia }\end{array}$ & Razón \\
\hline$\triangle$ Sexo & Moderadora & Género & Directo & Masculino y femenino & Nominal \\
\hline$\Delta$ Edad de cirugía & Moderada & Años & Indirecto & Años cumplidos & Razón \\
\hline$\Delta$ Tiempo hasta cirugía & Moderadora & $\begin{array}{l}\text { Meses entre } \\
\text { diagnóstico y cirugía }\end{array}$ & Directo & Número de meses & Razón \\
\hline $\begin{array}{l}\text { Número de focos } \\
\text { epileptógenos }\end{array}$ & Moderadora & Número & Indirecto & $\begin{array}{l}\text { - Único } \\
\text { - Múltiple }\end{array}$ & Nominal \\
\hline$\Delta$ Lado epileptógeno & Moderadora & Lado & Indirecto & $\begin{array}{l}\text { - Derecha } \\
\text { - Izquierda } \\
\text { - Bilateral }\end{array}$ & Nominal \\
\hline$\Delta$ Lóbulo epileptógeno & Moderadora & $\begin{array}{l}\text { Lóbulo (s) cerebrales } \\
\text { afectados }\end{array}$ & Indirecto & $\begin{array}{l}\text { - Temporal } \\
\text { - Frontal } \\
\text { - Multilobar }\end{array}$ & Nominal \\
\hline $\begin{array}{l}\text { Nivel cognitivo } \\
\text { prequirúrgico }\end{array}$ & Moderadora & $\begin{array}{l}\text { Coeficiente } \\
\text { intelectual }\end{array}$ & Indirecto & $\begin{array}{l}\text { - Normal (90-109) } \\
\text { - Medio bajo (80-89) } \\
\text { - Retraso mental leve (70-79) } \\
\text { - Retraso mental moderado (50-69) } \\
\text { - Retraso mental grave (20-49) }\end{array}$ & Ordinal \\
\hline $\begin{array}{l}\text { Número y dosis } \\
\text { de FAE }\end{array}$ & Moderadora & $\begin{array}{l}\text { Cantidad FAE y dosis } \\
\text { normales o altas }\end{array}$ & Indirecto & $\begin{array}{l}\text { - Dos a dosis habituales o elevadas } \\
\text { - Tres a dosis habituales o elevadas } \\
\text { - Cuatro a dosis habituales o altas }\end{array}$ & Razón \\
\hline$\triangle$ Tipo de cirugía & Moderadora & $\begin{array}{l}\text { Técnica quirúrgica } \\
\text { usada }\end{array}$ & Directa & $\begin{array}{l}\text { - Electrocorticografía de largo } \\
\text { plazo + resección focal } \\
\text { - ECoG intraoperatoria + } \\
\text { lobectomía temporal } \\
\text { - Hemisferectomía funcional }\end{array}$ & Nominal \\
\hline$\Delta$ Diagnóstico definitivo & Moderadora & $\begin{array}{l}\text { Características } \\
\text { clínicas, EEG y } \\
\text { patología }\end{array}$ & Indirecto & $\begin{array}{l}\text { - Displasia cortical focal } \\
\text { - Esclerosis del hipocampo } \\
\text { - Encefalitis de Rasmussen }\end{array}$ & Nominal \\
\hline $\begin{array}{l}\text { Efectos adversos } \\
\text { de cirugía }\end{array}$ & Moderadora & $\begin{array}{l}\text { Trastornos } \\
\text { neurológicos } \\
\text { posquirúrgicos }\end{array}$ & Indirecta & $\begin{array}{l}\text { - Ausente } \\
\text { - Transitoria } \\
\text { - Definitiva }\end{array}$ & Ordinal \\
\hline $\begin{array}{l}\text { Tiempo de seguimiento } \\
\text { posquirúrgico }\end{array}$ & Moderadora & Meses transcurridos & Directa & $\begin{array}{l}\text { Número de meses desde cirugía } \\
\text { hasta el último control }\end{array}$ & Razón \\
\hline
\end{tabular}

- Epilepsia refractaria: epilepsia que no logra el control de crisis con el uso de dos anticonvulsivantes adecuadamente elegidos a dosis adecuadas.
- Hospital Nacional Guillermo Almenara Irigoyen: sede central de la RAA, en los departamentos de Neurocirugía, Pediatría, Centro Quirúrgico y servicios de Psicología y Medicina Nuclear. 


\section{Indicadores de las variables}

- Eficacia: se estableció según la escala de Engel modificada; ${ }^{10}$ disminución porcentual de la frecuencia de crisis epilépticas antes y después de la cirugía.

$\triangle$ Edad de inicio de epilepsia: se determinó en forma directa según el número de años cumplidos al inicio de la primera crisis epiléptica.

$\Delta$ Sexo: se estableció en forma directa por el género de la paciente.

$\Delta$ Edad de cirugía: se estableció según el número de años cumplidos del paciente al momento de la cirugía definitiva de epilepsia.

^ Tiempo hasta la cirugía: se establecerá según el número de años transcurridos entre el inicio de la crisis y la fecha de la cirugía definitiva de epilepsia.

\ Número de focos epileptógenos: se establecerá según el informe de VEEG la cantidad de focos epileptógenos para inicio de eventos ictales.

- Lado epileptógeno: se establecerá según el Informe de VEEG, el hemisferio del foco epileptógeno del inicio de los eventos ictales.

^ Lóbulo epileptógeno: se estableció según el Informe de VEEG, el lóbulo comprometido en el inicio de los focos ictales.

- Nivel cognitivo prequirúrgico: según el informe de neuropsicología el valor de coeficiente intelectual y luego se establecerán las categorías de normal, retardo mental leve, retardo mental moderado y retardo mental grave.

- Número y dosis de FAE preoperatorios: según la historia clínica se determinarán el número de fármacos antiepilépticos antes de la cirugía definitiva y si la dosis correspondiente es la usual o superior en algunos de los fármacos.

^ Tipo de cirugía: se determinó directamente según el tipo de procedimiento establecido en el informe operatorio.

^ Diagnóstico definitivo: se estableció según los datos de la historia clínica, los informes de neuroimágenes estructurales y funcionales, informes de electrofisiología del VEEG y el informe final de anatomía patológica.
- Efectos adversos de la cirugía: se estableció según la historia clínica, la presencia o ausencia de efectos adversos que provocaron el aumento del número de días de hospitalización, la aparición de un defecto neurológico no presente al ingreso y presente al alta, con desaparición en los controles.

- Tiempo de seguimiento posquirúrgico: se estableció según la historia clínica el número de meses de seguimiento desde la fecha de cirugía definitiva de epilepsia hasta el último control.

\section{Muestra}

- Selección de la muestra: no aleatoria por conveniencia, según la demanda de los pacientes.

- Tamaño muestral: se ha estimado en cinco pacientes, dada la elevada respuesta obtenida.

\section{Materiales y métodos}

En los cinco pacientes referidos de neuropediatría y neurología tanto del HNGAl, como de los hospitales de la RAA, se siguieron los siguientes pasos:

$\Delta$ Evaluación clínica en las especialidades de neurología, neuropediatría y neurocirugía.

$\Delta$ Resonancia magnética en protocolo de epilepsia.

^ Tomografía de emisión de fotón simple.

凶 Tomografía de emisión de protones.

$\Delta$ Evaluación neuropsicológica.

$\triangle$ Resonancia magnética funcional.

$\triangle$ VEEG de 24 a 48 horas.

$\triangle$ Junta médica multidisciplinaria del equipo de epilepsia.

- Junta médica multidisciplinaria de neurocirugía.

- Revisión de historias clínicas y entrevistas a los padres.

- Tabulación y procesamiento de datos.

$\Delta$ Elaboración del informe final.

Técnicas aplicadas en la recolección de la información

El proceso de obtención de información para el estudio fue según las siguientes técnicas: revisión de historia clínica; revisión de los informes y las imágenes de resonancia y las imágenes funcionales 
de SPECT, PET y RM funcional; revisión de informes y registro de VEEG de 24 a 48 horas; revisión de informe de neuropsicología; revisión de informe operatorios, epicrisis, evoluciones posquirúrgicas ambulatorias; revisión de los informes de anatomía patológica.

\section{Instrumentos de medición}

Se utilizaron resonancia magnética (RM), RM funcional (RMf), tomografía de emisión de fotón simple (SPECT), tomografía de emisión de positrones (PET), test de Wada y VEEG prolongado

Análisis de datos

Se empleó la estadística descriptiva.

\section{RESULTADOS}

\section{Características demográficas}

Se realizó el estudio multidisciplinario y el tratamiento quirúrgico en siete pacientes con criterios de ER, con un promedio de edad al momento de la cirugía de 11,49 años (rango entre 3,4 y 35 años), de los cuales seis fueron mujeres y un paciente varón.

\section{Características clínicas}

La descripción de los resultados está presentada en la Tabla 2.

Las imágenes de los estudios de resonancia preoperatoria, PET o SPECT y EEG ictal se muestran en las Figuras 1, 2 y 3, respectivamente.

Los siete pacientes estudiados tuvieron una edad de inicio promedio de la epilepsia de 3,83 años (rango de 8 meses a 8 años).

El tiempo promedio de epilepsia previo a la cirugía definitiva de 7,38 años (rango de 1,25 a 28 años). Aunque este valor baja a 3,94 años al excluir el valor mayor.

La localización del origen de la epilepsia fue:

$\Delta$ En tres casos, encontramos epilepsia difusa hemisférica, dos del lado izquierdo y uno del lado derecho.

- En dos casos encontramos foco temporal, en uno bilateral y en el otro temporal derecho.

$\Delta$ En un caso el foco fue frontal bilateral. $\triangle$ En un caso encontramos focos bilaterales independientes a predominio izquierdo.

El nivel cognitivo prequirúrgico fue en dos casos medio bajo, dos con retraso mental leve y tres con retraso mental moderado.

El número de $\mathrm{FAE}$ utilizados en el preoperatorio fue de 2,86 (rango de 1 a 5 FAE).

\section{Resultados clínicos}

La eficacia de la cirugía en el control de la ER fue excelente en $71,4 \%$ (5 de 7 casos) con valores de escala de Engel I (desaparición de las crisis epilépticas) y buena en $28,6 \%$ ( 2 de 7 pacientes) con valores de escala de Engel II (disminución de crisis entre $75 \%$ y $99 \%$ ).

Los tipos de cirugía realizada fueron hemisferectomía funcional (3), lobectomía temporal lateral y mesiotemporal con electrocorticografía (2), resección multifocal guiada por electrocorticografía y callosotomía completa (1) e implantación de electrodos subdurales de largo plazo y resección cortical frontal en una segunda cirugía (1). La hemisferectomía funcional fue izquierda en dos casos del lado izquierdo, por encefalitis de Rasmussen, y derecha en un caso, por displasia hemisférica derecha.

Los efectos adversos posquirúrgicos permanentes se presentaron en $28,6 \%$ ( 2 de 7 casos): hemiparesia leve (1) y hemiparesia leve y afasia nominal parcial (1). En $71,4 \%$ (5 de 7 casos) no hubo ningún efecto adverso a largo plazo.

El resultado definitivo confirmado con patología fue encefalitis de Rasmussen (2), displasia cortical (3), esclerosis tuberosa (1) y esclerosis del hipocampo (1). El tiempo de seguimiento posquirúrgico fue de 22,4 meses (rango: 5-40).

\section{DISCUSIÓN}

Es la primera serie de siete casos de ER del HNGAl, estudiados en forma multidisciplinaria y con neuroimágenes estructurales y funcionales, con registro prolongado de actividad electroencefalográfica con video sincronizado con manejo quirúrgico. El cual muestra resultados exitosos con $71,4 \%$ de pacientes libres de crisis y el restante $28,6 \%$ con disminución de la frecuencia de 
crisis en $80 \%$, lo que demuestra la capacidad de nuestro equipo humano de realizar el manejo de estas patologías tan complejas y frecuentes con resultados similares a los obtenidos en centros de epilepsia del extranjero, tal como ha sido revisado en el resumen de evidencia de la cirugía de epilepsia hecha por el grupo de Calidad en la Salud de Ontario Canadá que describe una eficacia de $43 \%$ a $75 \%{ }^{9}$

El género marcadamente predominante fue el femenino (86\%), a diferencia de las publicaciones previas en las que no se evidencia predominancia de sexo alguno, ${ }^{11}$ en algunas crisis de ausencia se encuentra predominio femenino. La otra explicación es que al ser una serie pequeña de pacientes para el estudio de género mostraría resultados no representativos.

La edad de inicio de epilepsia fue en todos los casos de 3,83 años o menos, de los cuales tres casos iniciaron antes del año y medio de vida, al ser de origen congénito, los otros casos fueron a partir de los 4 años, en los casos de Encefalitis de Rasmussen los pacientes tuvieron 4 y 8 años que coinciden con el promedio de 6 años descritos en la bibliografía. ${ }^{12}$

Respecto al tiempo transcurrido entre el inicio de la epilepsia y el tratamiento quirúrgico definitivo fue relativamente largo en promedio (7,38 años), debido a que un caso demoró 28 años en ser referido. Si excluimos dicho caso, el promedio

Tabla 2. Resultados de estudio multidisciplinario y cirugía de la epilepsia refractaria HNGAI

\begin{tabular}{|c|c|c|c|c|c|c|c|}
\hline Variable & Caso 1 & Caso 2 & Caso 3 & Caso 4 & Caso 5 & Caso 6 & Caso 7 \\
\hline$\Delta$ Eficacia (Engel) & I & I & II & ॥ & I & I & I \\
\hline$\Delta$ Edad de inicio (años) & 8 & 4 & 0,75 & 5 & 7 & 0,66 & 1,4 \\
\hline$\Delta$ Sexo & Femenino & Femenino & Femenino & Femenino & Masculino & Femenino & Femenino \\
\hline$\triangle$ Edad de cirugía & 6 & 10 & 4 & 14 & 35 & 8 & 3,4 \\
\hline $\begin{array}{l}\text { Tiempo hasta } \\
\text { cirugía (años) }\end{array}$ & 1,25 & 1,33 & 3,08 & 9 & 28 & 7 & 2 \\
\hline $\begin{array}{l}\text { Número de focos } \\
\text { epileptógenos }\end{array}$ & Difuso & Difuso & Dos & Uno & Uno & Multifocal & Multifocal \\
\hline$\Delta$ Lado epileptógeno & Izquierdo & Izquierdo & Bilateral & Izquierdo & Derecho & Bilateral & Derecho \\
\hline $\begin{array}{l}\text { Lóbulo } \\
\text { epileptógeno }\end{array}$ & Hemisférico & Hemisférico & Temporal & Frontal & Temporal & Multilobar & Multilobar \\
\hline $\begin{array}{l}\text { Nivel cognitivo } \\
\text { prequirúrgico }\end{array}$ & $\begin{array}{l}\text { Rm leve } \\
\text { moderado }\end{array}$ & $\begin{array}{l}\text { Rm leve } \\
\text { bajo }\end{array}$ & $\begin{array}{l}\mathrm{Rm} \\
\text { bajo }\end{array}$ & $\begin{array}{l}\text { Rm medio } \\
\text { moderado }\end{array}$ & $\begin{array}{l}\text { Rm medio } \\
\text { moderado }\end{array}$ & $\mathrm{Rm}$ & $\mathrm{Rm}$ \\
\hline$\triangle$ Número y dosis $F A E^{\star}$ & 4 & 5 & 3 & 2 & 2 & 3 & 1 \\
\hline $\begin{array}{l}\text { Efectos adversos } \\
\text { posquirúrgicos }\end{array}$ & $\begin{array}{l}\text { Hemiparesia } \\
\text { y afasia }\end{array}$ & Hemiparesia & Ninguno & Ninguno & Ninguno & Ninguno & Ninguno \\
\hline$\triangle$ Tipo de cirugía & $\begin{array}{l}\text { Hemisferectomía } \\
\text { funcional }\end{array}$ & $\begin{array}{l}\text { Hemisferectomía } \\
\text { funcional }\end{array}$ & $\begin{array}{c}\text { Lobectomía } \\
\text { temporal } \\
\text { izquierda }\end{array}$ & $\begin{array}{c}\text { Corticectomía } \\
\text { frontal en } \\
2 \text { tiempos }\end{array}$ & $\begin{array}{c}\text { Lobectomía } \\
\text { temporal } \\
\text { izquierda }\end{array}$ & $\begin{array}{l}\text { Lesionectomía } \\
\text { múltiple + }\end{array}$ & $\begin{array}{l}\text { Hemisferectomía } \\
\text { funcional } \\
\text { callosotomia }\end{array}$ \\
\hline $\begin{array}{l}\text { Diagnóstico } \\
\text { definitivo }\end{array}$ & $\begin{array}{l}\text { Encefalitis de } \\
\text { Rasmussen }\end{array}$ & $\begin{array}{l}\text { Encefalitis de } \\
\text { Rasmussen }\end{array}$ & $\begin{array}{l}\text { Displasia } \\
\text { temporal }\end{array}$ & $\begin{array}{l}\text { Displasia } \\
\text { frontal }\end{array}$ & $\begin{array}{c}\text { Esclerosis } \\
\text { hipocámpica }\end{array}$ & $\begin{array}{l}\text { Esclerosis } \\
\text { tuberosa }\end{array}$ & $\begin{array}{l}\text { Displasia } \\
\text { hemisférica }\end{array}$ \\
\hline $\begin{array}{l}\text { Seguimiento } \\
\text { posquirúrgico (meses }\end{array}$ & es) & 40 & 31 & 19 & 17 & 5 & 5 \\
\hline $\begin{array}{l}\text { Número de crisis } \\
\text { pre/poscirugía }\end{array}$ & $\begin{array}{l}\text { Crisis parciales } \\
\text { continuas } / 0\end{array}$ & $\begin{array}{c}\text { Crisis parciales } \\
\text { continuas/0 }\end{array}$ & $\begin{array}{c}14 / 3 \\
\text { semanales }\end{array}$ & $\begin{array}{c}3 / 0,5 \\
\text { semanales }\end{array}$ & $\begin{array}{c}2 / 0 \\
\text { semanal }\end{array}$ & $\begin{array}{c}1 / 0 \\
\text { semanal }\end{array}$ & $\begin{array}{c}7 / 0 \\
\text { semanal }\end{array}$ \\
\hline
\end{tabular}




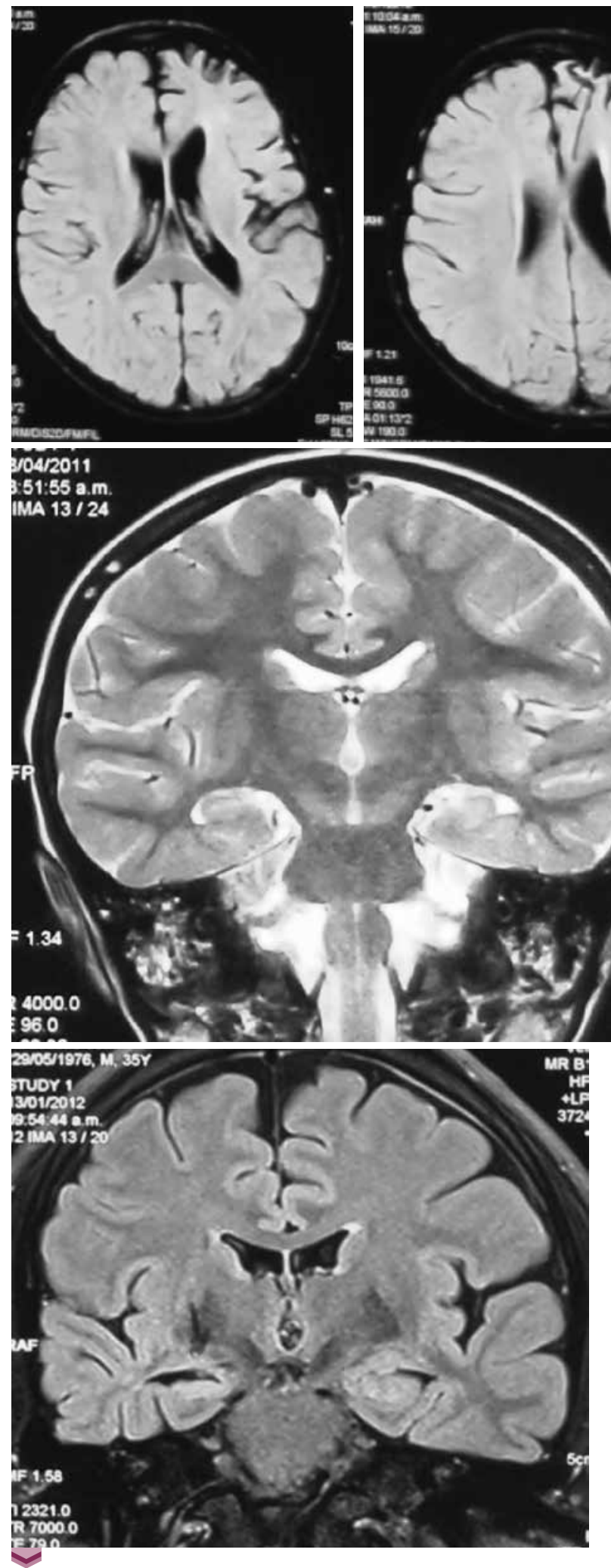

de tiempo entre el inicio de las crisis y la cirugía fue de 3,94 años, el cual es similar al de otras publicaciones internacionales. ${ }^{13}$

Al analizar la localización del foco o focos epileptógenos, estos han correspondido a los diferentes tipos de patología intervenidos: En los dos casos con encefalitis de Rasmussen fueron hemisféricos del lado izquierdo, Un caso con displasia severa del hemisferio derecho, en los dos casos de displasia cortical fueron uno en el lóbulo

Figura 1. Caso 1. RM en modo FLAIR con hipotrofia del hemisferio izquierdo y dilatación ventricular ipsilateral. Caso 2: Resonancia Magnética en modo FLAIR, con hiperintensidad en hemisferio izquierdo. Caso 3: RM coronal modo T2 con hipotrofia mesiotemporal izquierda. Caso 4. RM en modo FLAIR del caso 4 con calcificación frontoporal derecha e hiperintensidad subcortical con zonads de displasia cortical frontopolar bilateral. Caso 5. RM en modo FLAIR coronal con hipotrofia de región mesiotemporal derecha. Continúa ... 

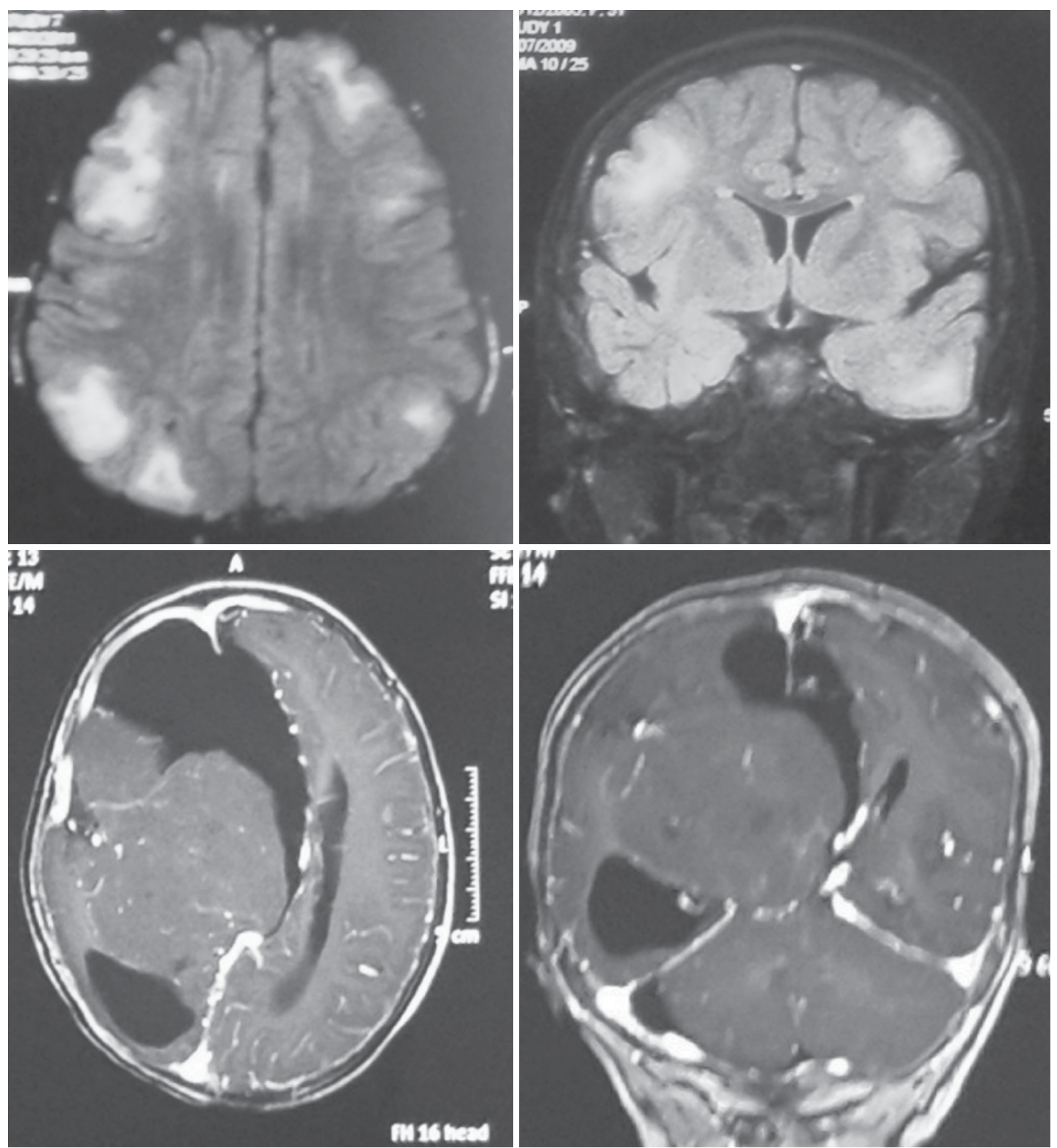

... continuación Figura 1. Caso 6. RM FLAIR coronal y axial con múltiples nódulos hiperintensos sugestivos de esclerosis tuberosa. Caso 7. RM coronal y axial con displasia severa de hemisferio derecho.

frontal izquierdo, un caso con esclerosis tuberosa múltiple bilateral independientes y el otro caso temporal bilateral y en el último caso de esclerosis del hipocampo en el lóbulo temporal derecho, esta distribución en los cuatro primeros casos es similar a otra publicación de ubicación por SPECT y RM corregistrada con VEEG ${ }^{14}$
El nivel cognitivo preoperatorio de los pacientes fue menor al normal en todos los casos intervenidos, debido a la interferencia que producen las crisis en el desarrollo cerebral normal de los niños operados, tal como se ha publicado en otras series que muestran compromiso en $89 \%$ de los casos, ${ }^{15}$ en nuestra serie también se ha observado que el 


\section{Anta Médica Peruana}
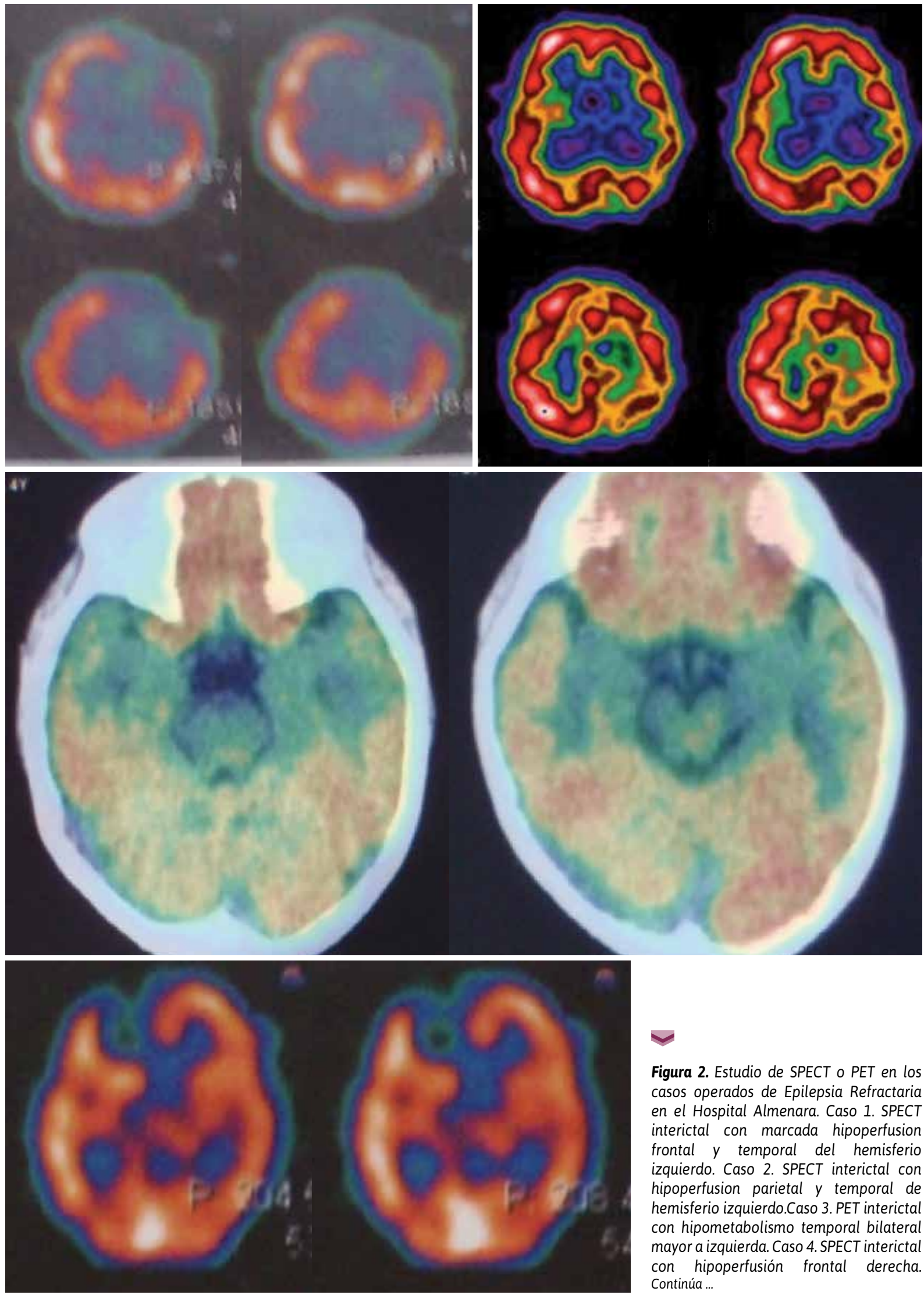

$\checkmark$

Figura 2. Estudio de SPECT o PET en los casos operados de Epilepsia Refractaria en el Hospital Almenara. Caso 1. SPECT interictal con marcada hipoperfusion frontal y temporal del hemisferio izquierdo. Caso 2. SPECT interictal con hipoperfusion parietal y temporal de hemisferio izquierdo.Caso 3. PET interictal con hipometabolismo temporal bilateral mayor a izquierda. Caso 4. SPECT interictal con hipoperfusión frontal derecha. Continúa ... 


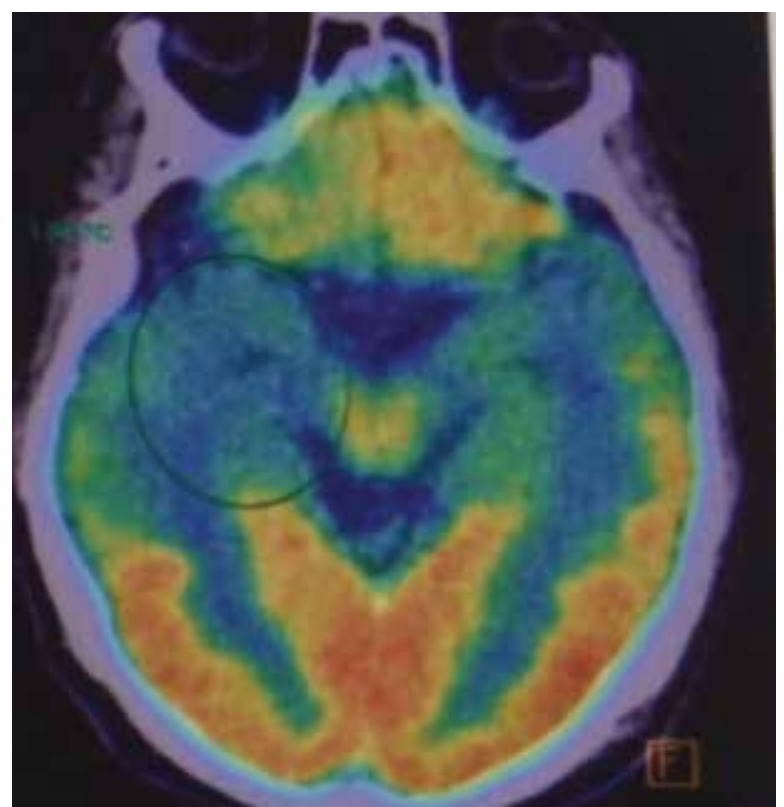

grado de gravedad del compromiso cognitivo está relacionado a la duración de la epilepsia y la frecuencia de las crisis.

En los casos operados, se encontró una relación directa entre la frecuencia de crisis en el preoperatorio y el número de fármacos antiepilépticos usados, esta relación ya ha sido descrita en publicaciones previas. ${ }^{16}$

Los efectos adversos permanentes se han presentado en $14 \%$ de los casos operados y solo en los

... Continuación Figura 2. Caso 5. PET con hipometabolismo severo mesiotemporal derecho. Caso 6. ET con áreas hipometabólicas bilaterales frontales, temporales bilaterales y occipital derecha. Caso 7. PET interictal con hipometabolismo hemisférico e hipermetabolismo focal.
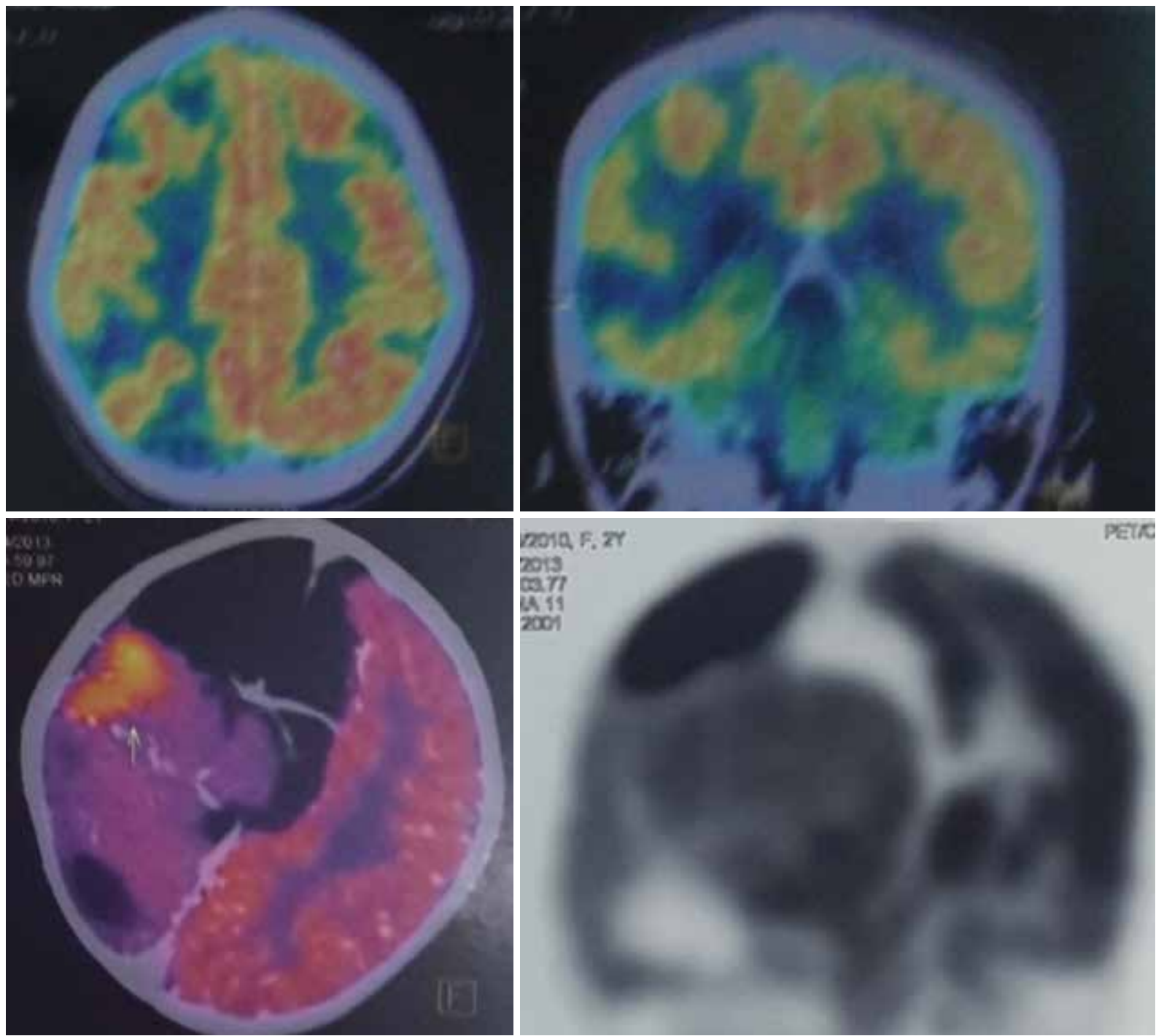


\section{Anta Méílica Pernana}

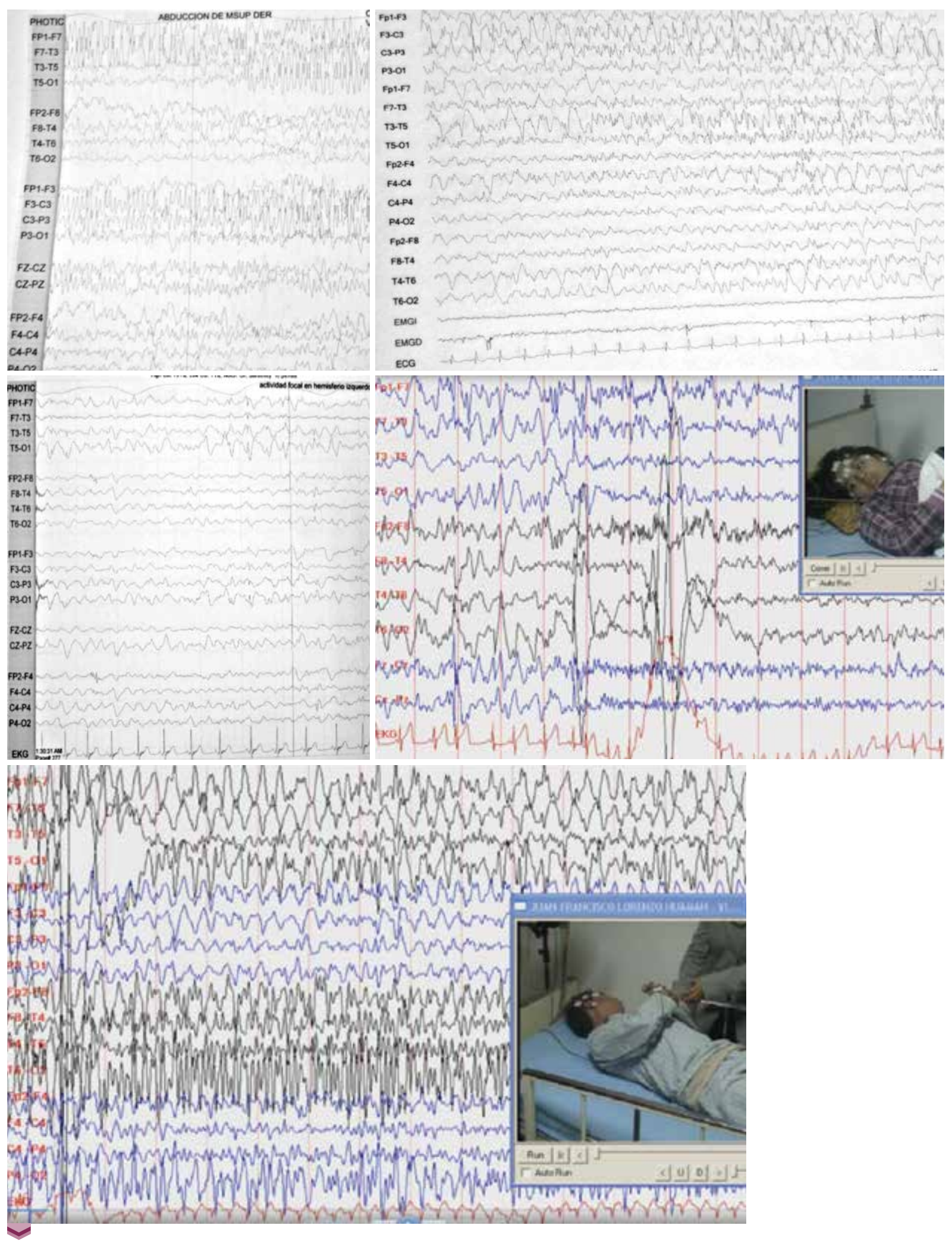

Figura 3. Estudios de EEG ictal de los casos operados de epilepsia refractaria en el Hospital Almenara. Caso 1. EEG del inicio ictal en las regiones frontal y temporal izquierda. Caso 2. EEG del inicio ictal en la region central frontoparietal izquierda. Caso 3. EEG de inicio ictal en region temporal izquierda. Caso 4. Video-EEG con inicio de crisis frontal izquierda con rotación de cabeza a derecha y desviación de la mirada a derecha. Caso 5. Video-EEG que muestra inicio de crisis temporal derecha con rotación de cabeza y cuerpo a izquierda. Continúa ... 


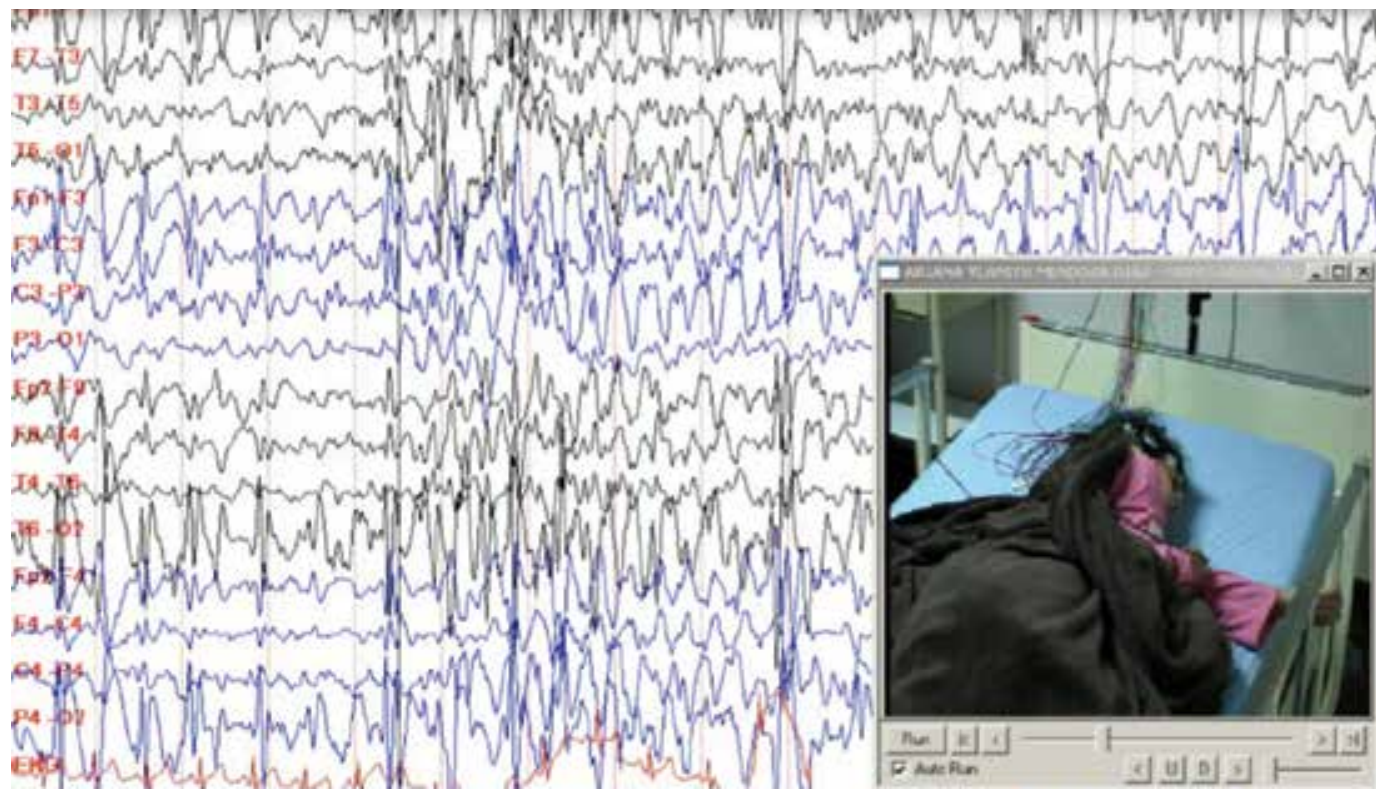

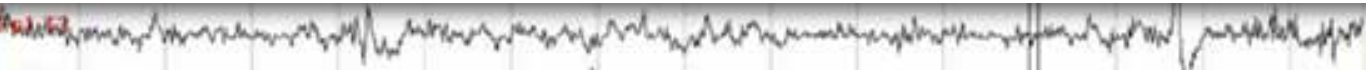

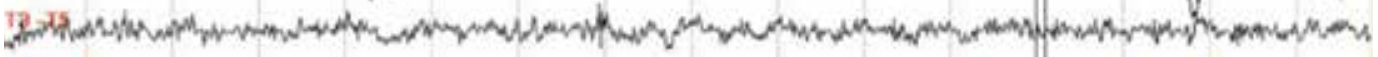

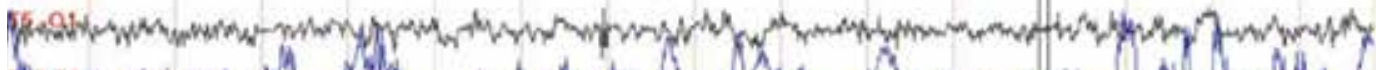

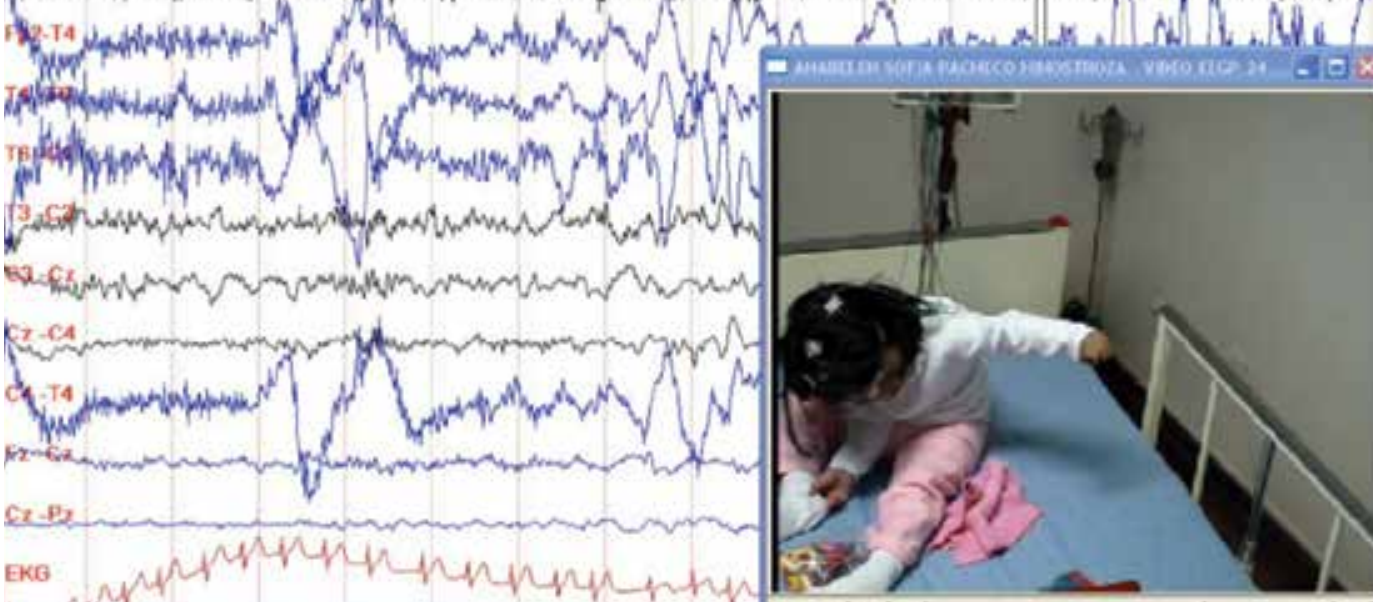

... Continuación Figura 3. Caso 6. Video-EEG que muestra inicio de crisis con descargas bitemporales y frontal izquierda con extensión del brazo izquierdo. Caso 7. Video-EEG con crisis de inicio fronto temporal y central derecho con extensión tónica del miembro superior izquierdo.

sometidos a hemisferectomía funcional del lado izquierdo, con hemiparesia leve a predominio braquial distal, que les permite caminar, y en el caso de mayor edad (10 años) presentó afasia parcial de expresión. Lo que ratifica que los efectos adversos son menores o nulos cuanto más antes sea la cirugía y en especial si es de hemisferio dominante.

El tipo de cirugía realizado fue según la localización y la extensión de los focos epileptógenos utilizados. En la epilepsia frontal izquierda fue necesario realizar la cirugía en dos tiempos: uno para implantar los electrodos subdurales de largo plazo y el otro para la resección respectiva. En los otros seis casos, la cirugía se hizo en un solo procedimiento. En los tres casos con hemisferectomía no se hizo registro alguno intraoperatorio y en los dos casos con epilepsia temporal se utilizó la ECoG IO. En la paciente con esclerosis tuberosa se hizo lesionectomía guíada por ECoG IO y complementada con callosotomía. 
Los resultados de anatomía patológica fueron en tres casos displasia cerebral corticosubcortical, en dos casos encefalitis de Rasmussen, un caso con esclerosis tuberosa y en un caso esclerosis del hipocampo. Estos resultados corresponden a la evolución clínica y la respuesta a la cirugía.

\section{CONCLUSIONES Y RECOMENDACIONES}

El estudio multidisciplinario y el tratamiento quirúrgico es eficaz en el manejo de la epilepsia refractaria (ER) en los pacientes del Hospital Nacional Guillermo Almenara. La evaluación con neuroimágenes de resonancia magnética encefálica, PET scan, SPECT, evaluación neuropsicológica y videoencefalograma son útiles para identificar el origen de los focos epileptógenos en los casos de ER. La displasia cortical y la encefalitis autoinmune son las causas más frecuentes de ER en los niños intervenidos en nuestra serie. A mayor edad de la hemisferectomía funcional se presenta una menor recuperación funcional, por lo que es importante el manejo precoz.

Se recomienda establecer una unidad orgánica para el manejo de la epilepsia refractaria en la Red Asistencial Almenara, con un sistema adecuado de referencia y con al menos dos ambientes de video-EEG. Se recomienda realizar un programa de capacitación y difusión del manejo de la epilepsia en todos los niveles de atención de EsSalud para la identificación y referencia precoz de los pacientes con epilepsia refractaria. Se recomienda establecer un programa de difusión, promoción y educación a la población sobre la identificación y atención adecuada de la epilepsia.

\section{REFERENCIAS BIBLIOGRÁFICAS}

I. Langan Y, Nashef L, Sander J. Certification of deaths attributable to epilepsy. Short Report. J Neurol Neurosurg Psychiatry. 2002;73(6):75I-752.

2. Marino Jr.R, Cukiert Arthur, Pinho E. Aspectos epidemiológicos da epilepsia em São Paulo: um estudo da prevalência. Arq. NeuroPsiquiatr. 1986;44(3):243-254.

3. Villarán MVI, Montano SM, Gonzalvez G, Moyano LM, Chero JC, Rodriguez S, et al; Cysticercosis Working Group in Peru. Epilepsy and neurocysticercosis: an incidence study in a Peruvian rural population. Neuroepidemiology. 2009;33(I):25-3I.

4. United States Census Bureau, International Data Base [Internet]. Washington, DC:World Population [citado 5 may 2013]. Disponible en:www.census.gov/popclock

5. World Health Organization. Epilepsy [Internet]. Geneva,Switzerland: WHO;2012 [citado 5 mayo 2013]. Disponible en: http://www.who. int/mediacentre/factsheets/fs999/en/index.html

6. World Health Organization, Department of Mental Health and Substance Abuse, Programme for Neurological Diseases and Neuroscience; ILAE; IBE. Atlas - Epilepsy Care in the World 2005. Geneva, Switzerland:WHO; 2005. Disponible en: http://www.who. int/mental_health/neurology/Epilepsy_atlas_rl.pdf

7. International League Against Epilepsy (ILAE) Commission Report. The epidemiology of the epilepsies: future directions. Epilepsia. 1997;38(5):6|4-6|8.

8. Instituto Nacional de Estadística e Informática - INEI, Perú: Estimaciones y Proyecciones de Población 1950-2050. Boletín de Análisis Demográfico N 35. Lima: INEl; 200 I.p. 48.
9. Health Quality Ontario. Epilepsy surgery:an evidence summary. Ont Health Technol Assess Ser [Internet]. 2012;12(I7): I-28. Disponible en: http://www.hqontario.ca/en/documents/eds/2012/full-reportepil-surg.pdf

10. Engel J Jr, Van Ness PC, Rasmussen TB. with respect to epileptic seizures. In:J Engel Jr ed. Surgical Treatment of the Epilepsies. 2nd ed. New York: Raven Press; 1993. p. 609-62I.

I I. Berg AT. Epidemiology of the intractable generalized epilepsies. En: Lüders HO, editor. Epilepsy surgery. London: Informa healthcare; 2008. p. 207-2I4.

12. Bien CG, Granata T,Antozzi C, Cross JH, Dulac O, Kurthen M, et al. Pathogenesis, diagnosis and treatment of Rasmussen encephalitis: a European consensus statement. Brain. 2005; I 28(Pt 3):454-47I.

13. Berg AT, Langfitt J, Shinnar S, Vickrey BG, Sperling MR, Walczak T, et al. How long does it take for partial epilepsy to become intractable? Neurology. 2003;60(2):186-190.

14. Miśko J, Jurkiewicz E, Bekiesińska-Figatowska M, Kamińska $A$, Bachański Z, et al. Usefulness of corregistration and post-processing of MR and interictal SPECT images for localization of epileptogenic focus in children - preliminary report. Pol J Radiol. 201 I;76(4):7-14.

15. Ramantani G, Kadish NE, Strobl K, Brandt A, Stathi A, Mayer H, et al. Seizure and cognitive outcomes of epilepsy surgery in infancy and early childhood. Eur J Paediatr Neurol. 2013;17(5):498-506.

16. Salih MR, Bahari MB, Hassali MA, Shafie AA,AI-Lela OQ,Abd AY, et al. Characteristics of seizure frequency among Malaysian children diagnosed with structural-metabolic epilepsy. J Neurosci Rural Pract. 2012;3(3):244-250.

\section{Correspondencia}

Dr. Marco Antonio Mejía-Tupa marcomejiatupa@yahoo.com

Fecha de recepción: 13 de setiembre de 2014

Fecha de aceptación: 25 de setiembre de 2014

\section{Conflictos de interés}

El autor declara no tener conflictos de interés durante el planteamiento, ejecución de la investigación y la elaboración del artículo para su publicación. 